\title{
RESP: Relay Suitability-based Routing Protocol for Video Streaming in Vehicular Ad Hoc Networks
}

\author{
O.A. Hammood, N. Nizam, M. Nafaa, W.A. Hammood
}

\author{
Omar A. Hammood*, Mohd Nizam, \\ Muamer Nafaa, Waleed A. Hammood \\ Department of Computer System and Software Engineering \\ University Malaysia Pahang (UMP), Malaysia \\ Kuantan-26300, Pahang, Malaysia \\ *Corresponding author: omer_almajeed@yahoo.com \\ mnizam@ump.edu.my, Muamer.scis@gmail.com, engwaleed54@yahoo.com
}

\begin{abstract}
Video streaming in Vehicular Ad Hoc Networks (VANETs) is a fundamental requirement for a roadside emergency and smart video surveillance services. However, vehicles moving at a high speed usually create unstable wireless links that drop video frames qualities. In a high-density network, network collision between vehicles is another obstacle in improving the scalability of unicast routing protocols. In this paper, the RElay Suitability-based Routing Protocol (RESP) which makes a routing decision based on the link stability measurement was proposed for an uninterrupted video streaming. The RESP estimates the geographic advancement and link stability of a vehicle towards its destination only in the small region. To ensure the reliability while extending the scalability of routing, the relay suitability metric integrates the packet delay, collision dropping, link stability, and the Expected Transmission Count (ETX) in the weighted division algorithm, and selects a high-quality forwarding node for video streaming. The experimental results demonstrated that the proposed RESP outperformed the link Lifetime-aware Beacon-less Routing Protocol (LBRP) and other traditional geographical streaming protocols in providing a high packet delivery ratio and less packet delay with various network densities, and proved the scalability support of RESP for video streaming.

Keywords: Video streaming, scalability, geographic unicast routing, link stability, reliability, QoS.
\end{abstract}

\section{Introduction}

Vehicular Ad Hoc Networks (VANETs) are widely applied in information and communication technology to ease transportation problems $[8,23]$. Video streaming in VANETs is a central part towards the realization of intelligent transportation systems $[2,31]$, and it has many promising potential applications, ranging from safety to entertainment. However, video streaming in VANETs faces several challenges due to the stringent video quality level requirements $[7,9]$. The major problems associated with scalable video streaming over VANETs are frequent link failures [33] due to greedy vehicle mobility, lack of link quality measurement, and link lifetime measurement in terms of the vehicle velocity rather than the relative velocity of vehicles. The network condition is dynamic with respect to vehicle densities and the distance between the source and the destination vehicle [22]. The existing geographic routing protocols based on greedy or improved greedy routing determine the shortest routing paths in such a way that it can be scaled up to a large VANET environment; however, it degrades the performance of the routing approach under high mobility scenarios. The integration of different routing aspects such as relative velocity, densitybased collision probability, distance to reach the destination, and Expected Transmission Count (ETX) [15] in the measurement of routing relay suitability can improve the performance of video streaming over the dynamic VANET environment. An improper integration of these parameters 
tends to compensate each other for their influence on the scalable and efficient router selection for video streaming [4]. To cope with these challenges, the geographic unicast routing protocols have been employed as a suitable solution for the distribution of video flows in VANETs [24].

Recently, several geographic unicast routing protocols have evolved to achieve scalability in video streaming, however, a scalable video streaming that meets the stringent video quality requirements without video distortions under a highly dynamic network topology is a challenging task in unicast routing. Greedy Perimeter Stateless Routing (GPSR) is a commonly used geographic unicast routing protocol in wireless networks. The conventional location-based routing protocols depend on the distance metric in which the nearest vehicle is selected as a forwarder. In highly dynamic networks, the greedy node selection can lead to frequent link failures and retransmissions. Beside the dynamic network topology, video streaming solution has to comply with the Quality of Service (QoS) requirements [25]. To decide the reliable and QoS forwarding vehicle in the network, the recent geographic routing protocols must accumulate multiple routing factors.

Geographic unicast routing is more effective for a wireless communication [10,14], however, there are many issues in implementing a scalable and reliable geographic unicast routing over VANETs. For example, unicast geographic routing selects a greedy node in the communication range with the aim of advancing the packet forwarding [3]. Despite distance information, a common aspect of the geographic routing protocol is that it must select and relay data packet through a stable node. Two key facts that limit greedy routing performance in video streaming are node mobility and collision due to the unreliability of the greedy node. When a packet is dropped due to node mobility or collision, the sender node either retransmit the packet to the same forwarder or through the currently available greedy node. Consequently, it triggers unnecessary retransmissions, an end-to-end packet delay, and even video buffering and distortion. It is essential to focus on the trade-off between the suitability of a node in advancing packets toward the destination and the stability of its connectivity to the sender node.

In this paper, the RElay Suitability-based unicast routing Protocol (RESP) for an uninterrupted video streaming in the VANET topology is proposed with the aim of extending the scalability of geographic unicast routing protocols. The proposed RESP builds a small region at the boundary of the communication range using an offset angle and integrates the compensating factors using a weighted division algorithm to improve the performance of a VANET router selection process. It significantly reduces packet retransmission and propagation delay in video streaming.

The main contributions of the proposed RESP protocol are summarized as follows:

- The proposed RESP protocol considers multiple routing aspects for an efficient unicast routing and scalable video delivery with minimized distortions and buffering.

- Node density-aware data collision and relative velocity-aware link stability measurements facilitate the scaling up of RESP to a larger vehicular network.

- The geographical advancement of a near-by vehicle towards the destination is considered in the router selection to avoid the involvement of several intermediate vehicles in the routing. Further, the consideration of ETX avoids packet loss, resulting in a successful timely video frame delivery.

- The weighted division algorithm integrates multiple routing aspects and ensures an efficient and stable link selection for video delivery.

- The performance of the RESP routing protocol was evaluated using a network simulator and compared to the routing policy centralized in terms of packet delivery ratio (PDR), 
delay, overhead, and frame setback ratio (FSR).

The remaining part of this paper is organized as follows: Section 2 presents a discussion of the existing related works while section 3 presents the idea of the proposed system model, including the positive forwarding region formation, the degree of mobility, different routing aspects of relay suitability factor, forwarding probability and delay metrics, and scalable and QoS data forwarding by applying weighted division algorithm. In section 4, the performance of the proposed system was evaluated and presented while section 5 presents a conclusion and a brief introduction to the intended future work.

\section{Related works}

Several unicast routing protocols have been proposed for video streaming in VANETs [20]. The geographic unicast routing protocols are classified into three types, which are greedy routing, opportunistic routing, and trajectory-based routing protocols [5,19]. In VANETs, a commonly used routing strategy is the greedy forwarding strategy in which a node selects a neighbor close to the destination for data forwarding [26]. The opportunistic routing scheme follows the storecarry-and-forward technique and avoids unnecessary packet dropping especially when no forwarding node is available. However, it is prone to long delays and inadequate for video-streaming. The trajectory-based strategy provides more importance to a vehicle to act as a forwarder when it is moving towards the destination vehicle. The GPSR is a greedy-based unicast routing protocol in VANETs which assumes that the nodes are aware of their own position and the location of the destination [18]. The GPSR follow two different modes for data routing, which are the greedy mode and the perimeter mode. The perimeter mode is applied when there is no greedy router in the communication range. However, the greedy mode can fail since there is a high chance of the greedy router to move out of the communication range. Conventionally, several works have been presented on improving the basic GPSR [5,28]. The movement prediction routing (MOPR) considers the link stability concept and attempts to solve the issues in greedy routing mode while the improvement GPSR (I-GPSR) consolidates different metrics such as the distance to the destination, vehicle density, moving direction, and vehicle speed to make routing decisions. Likewise, the multimedia multimetric map-aware routing protocol (3MRP) [17] exploits the distance to the destination, vehicle density, trajectory, and available bandwidth to select the next hop for routing while the available bandwidth estimation (ABE) [1] calculates the available bandwidth on a link between two nodes. The MMMR exploits the roadmap considering the possible obstacles; however, it assigns an equal importance or weight to all the metrics in the score estimation. A better scheme must provide variable weights to the routing metrics depending on the current network conditions. However, the acquisition of motion prediction information is a difficult task. Most of the conventional routing techniques focus only on better packet delivery rates with low data delivery delays. However, they do not address the quality of video delivery when users are watching video sequences. Video dissemination techniques have not considered video qualities $[6,20]$ which is a crucial concept for the success of safety and traffic-warning video streaming in VANETs [18]. Thus, the multi-hop routing service is essential to the awareness of Quality of Experience (QoE) requirements and network conditions. It assists the video streaming approach to avoid the loss of video sequences and quality. A real-time video dissemination with a QoE-supportive solution in VANET [6] imposes the use of a distortion threshold. QoE represents the experience of the end users on a delivered service and their satisfaction rate about it. It considers the average delay in routing decisions, however, average delay reduction is not equivalent to QoE maximization [25]. The network conditions include collision warning, traffic congestion, and sharing parking information. When a collision or network congestion occurs, 
video streaming might be affected. The main reason behind network congestions and collision is a high routing overhead and to solve this issue, the beaconless routing protocols have been proposed $[20,21]$ Rather than a continuous information exchange with the neighboring vehicles, each vehicle considers the speed and position information before making any forwarding decision [11]. From the information, the receivers set a timer and the vehicle to first reach count zero will be selected as the next hop relay. For a high traffic collision environment, taking a routing decision based on the distance and vehicle stability is insufficient. The reactive routing protocols have a better mean opinion score in a VANET environment [27] whereas the location-based VANET routing protocols have a good result due to location accuracy [12,24]. However, they face a degradation in average mean opinion score [32] and affect the quality of video streaming over VANETs. Several works have focused on the performance evaluation of the position-based routing protocols for video streaming in VANETS based on the peak signal-to-noise ratio, video quality metric, and QoE measures [25,32]. To avoid the issue of retransmission, ETX has been employed in VANET protocols, however, ETX alone tends to present a long routing path and high delays. Thus, neither the location-based distance measure nor the ETX metric alone is efficient for video streaming over VANETs. To accomplish this, the proposed RESP protocol exploits relay suitability as a metric of communication quality and considered different routing aspects in relay suitability estimation.

\section{The proposed system model idea}

The proposed work considered the proximity of the vehicles to the destination. Expected Transmission Count (ETX), collision probability, and relative velocity based link stability in relay suitability measurement. The weighted division algorithm is used for an efficient integration of factors in the scalable router selection for unicast geographic routing. There are two different routing aspects considered in relay suitability measurement - data forwarding probability and data forwarding delay. The first aspect includes the link stability and collision probability. Figure 1 demonstrates the RESP architecture of the proposed work.

Considering the vehicle velocity alone is not feasible for scalable router selection because the velocity difference between two vehicles is dependent on the proximity of the vehicle. A considerable number of neighbors increase the possibility of packet losses due to collisions. A high traffic, to a greedy vehicle, results in collision and packet loss and limit the quality of a greedy link since it increases video distortion. Thus, the proposed work considered the collision probability in a scalable router selection. The second routing aspect handles the problem of data forwarding delay with the support of geographical advancement towards the destination and ETX. The proposed work only considered a few vehicles located in a preferable forwarding region (near to the destination vehicle). It avoids the selection of the number of hops to reach the destination and a high computational time. The sending vehicle applies the relay suitability measurement only on the vehicles in a preferable forwarding region. The distance routing metric alone enables the routing protocol to select the shortest routing path, however, it is prone to transmission failures due to poor link quality since the transmission capability of a node is not considered in the decision-making process.

FThe selection of routing vehicles with low ETX increases the reliability of a network. However, ETX alone is prone to long routing paths and high latency on routing video frames. Thus, the distance or ETX metric alone is inefficient for video streaming over VANETs. To accomplish this, the proposed work takes into account different routing aspects in relay suitability estimation. Moreover, simply combining the routing factors is not an adequate way, since these are compensating with each other. Thus, the proposed work designs an efficient weighted division algorithm and efficiently delivers the video stream over selected scalable routing vehicles. 


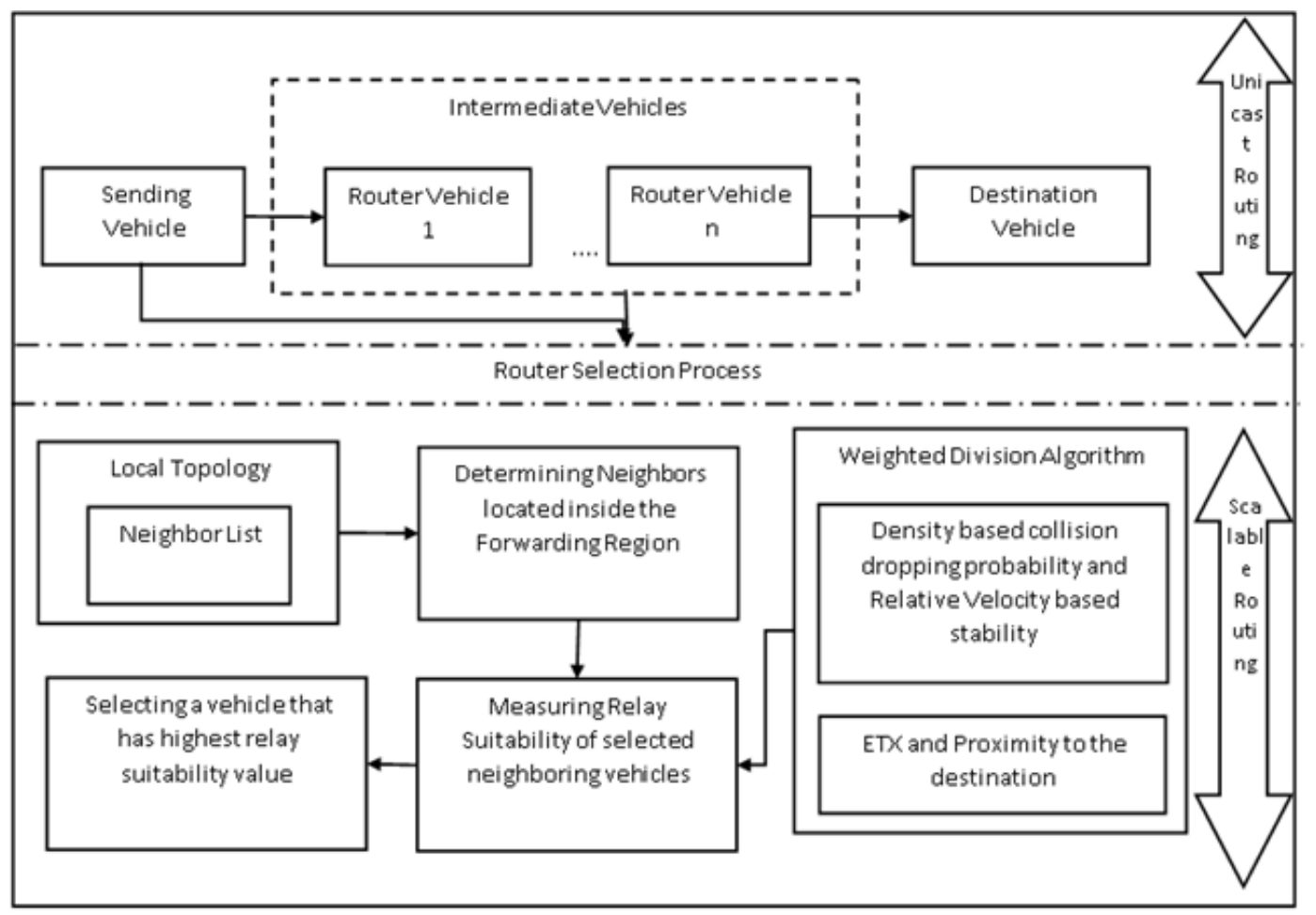

Figure 1: RESP architecture

\section{Proposed routing mechanism}

This section is intended to explain the mechanism of the proposed routing protocol and how this new mechanism can enhance the performance of the proposed protocol.

\subsection{Positive forwarding region formation}

The proposed work focused on the consolidation of multi-objective metrics which combine delay and stability with a successful forwarding probability. Delay and packet loss should not exceed their predefined limits since both are the deciding factors of video quality. The transmission of a video demands the use of high-quality links within a short time, thus, high-quality positive forwarding nodes must be selected from the neighbor list. Every node must broadcast a neighbor's discovery message to identify its neighboring nodes $(\mathrm{Nn})$ in the communication range $\mathrm{R}$. A node which receives the request message must respond by sending its identification number (ID) and the information relevant to the multi-objective metric. Every node receives several messages from its neighbors and builds its routing table. The nodes follow the periodic alive message propagation to maintain their presence in the communication range as a neighbor. If a node does not receive an alive message from the registered neighboring node until a predefined period, it erases the entry related to such neighbor from its routing table. Before selecting the router node, the source node $(\mathrm{Sn})$ executes the multi-objective function. The proposed work employs no execution control, the sender node would be executed for the neighboring nodes located in all the directions ad this results in an imperfect router selection and affects the video quality. Since there are compensating factors for distance metric, the sender selects and transmits the packet to a node in the backward direction to the destination. Therefore, to minimize the unnecessary transmission hop increment and quality degradation, each node that transmits the data packet must generate a "forwarders list" based on the distance information and apply the link quality 
measurement on them using a weighted division algorithm. The notations used in the work are listed in table 4.

Table 1: Notations used in RESP protocol

\begin{tabular}{|c|c|}
\hline Notations & Description \\
\hline $\mathrm{Nn}$ & Neighboring node \\
\hline $\mathrm{R}$ & Communication range \\
\hline $\mathrm{SN}$ & Source node \\
\hline Lsx, Lsy & Location Coordinates of node SN \\
\hline$\overline{\mathrm{DN}}$ & Destination node \\
\hline DistND & Distance between the destination and neighboring node \\
\hline DistSD & Distance between the source and destination node \\
\hline POSFN & Positive forwarding region \\
\hline ETX & Expected Transmission count \\
\hline$\Delta v_{j}$ & Relative velocity between node ${ }_{j}$ and ${ }_{i}$ \\
\hline $\mathrm{CP}_{j}$ & Collision probability of node ${ }_{j}$ \\
\hline NNM & The neighbors which are added in the MPFN of a node \\
\hline Link Quality $_{i, j}$ & Quality of a link ${ }_{i, j}$ \\
\hline$\alpha$ and $\beta$ & Weighting factors \\
\hline$P_{i, j}$ and $P_{j, i}$ & Weighting The packet delivery ratio of links ${ }_{i, j}$ and ${ }_{j, i}$ \\
\hline
\end{tabular}

Every forwarder of the data packets must generate a forwarders list. Note that the SN and intermediate nodes exploit the same process. To route towards the destination nodes (DN), the SN obtains the location information of the DN using the external location server. By applying Equation 1, the sender node estimates the distance between the source (Lsx, Lsy) and destination with the location coordinates ( $\mathrm{Ldx}$, Ldy). The DistND represents the distance between the destination and the neighboring nodes while DistSD represents the distance between the source and the destination nodes. From Equation 2, the sender node generates the set of forwarding nodes (FN), which include the nodes that satisfy the condition of DistSD > DistND.

$$
\begin{gathered}
\text { Dist_SD }=\left[\left(L_{-} d x-L_{-} s x\right)^{2}+\left(L_{-} d y-L_{-} s y\right)^{2}\right]^{(1 / 2)} \\
P S O_{F N}=\left\{F_{N} \mid N_{N} \in \operatorname{Dist}_{S D}>\text { Dist }_{N D}\right\}
\end{gathered}
$$

The process of forwarding node selection from the POSitive FN (POSFN) set provides the direction to deliver the data packets to the destination DN. In this way, although the data packets can approach the DN, the reachability cannot be guaranteed due to the negligence of the communication quality between the nodes. The proposed RESP needs to identify the quality routers that forward the data packets by considering the offset angle-based degree of mobility.

\subsection{Degree of mobility}

The proposed RESP balances the router suitability to forward the video packets between the node geographic advancement towards the destination and the link stability. To do this, the proposed RESP protocol considered a small region at the boundary of the communication range formed by the offset angle and the source node as the center of the circle. The restricted small region has been referred to as the "most preferable forwarding area" (MPFN) (Figure 2). Since the vehicles present at the edge of the communication range can move out of the communication range at any time, the vehicles present in the MPFN area were selected for the measurement 
of relay suitability. The preferable routers have a small degree of mobility. Also, there is a chance of experiencing a bad signal reception which can negatively impact the video quality. The forwarding region (FN) and the MPFN are shown in Figure 2

The area of the segment AreaTUV in a communication range of a node is named as the forwarding region, and the nodes located within the AreaTUV are denoted as a set of FN. The restricted segment area of Area ${ }_{T U V}$ is denoted as MPFN (Area $O P Q R$ ) because the nodes within the segment of the Area ${ }_{V O P}$ can move out of the communication range of a sender node at any time. This can increase the uncertainty of a forwarding node, thus, the RESP restricts the forwarding region segment area and selects a preferable forwarding region for routing. Notably, the nodes within the segments ofArea ${ }_{S Q R}$, Area ${ }_{S T O}$, and Area $_{S U P}$ increased the delay due to the long traveling path of a packet towards the destination. TheArea $O P Q R$ is estimated using the following equations:

$$
\text { Area }_{O P Q R}=\text { Area }_{S O P}-\text { Area }_{S Q R}
$$

The points 'O' and 'p' lie on the circle of communication range, with an angle of $\alpha$. Initially, the value of $\alpha$ is assigned as 450. If there are no nodes within the MPFN, all the nodes in the FN that satisfy the conditions of Equation 2 are involved in the relay suitability measurement. Moreover, points 'Q' and ' $Z$ ' lie at the distance of half of the communication range with an angle of $\alpha$.

$$
\begin{gathered}
\text { Area }_{S O P}=\left(\frac{\text { Dist }_{o p}}{4}\right) x\left(4 R^{2}-\text { Dist }_{o p}{ }^{2}\right)^{1 / 2} \\
\text { Area }_{S Q R}=\left(\frac{\text { Dist }_{Q R}}{4}\right) x\left(4 R^{2}-\text { Dist }_{Q R}{ }^{2}\right)^{1 / 2}
\end{gathered}
$$

The Area $O P Q R$ is estimated by substituting Equations 4 and 4 in 3. From the measured area, the nodes present inside the area are called the preferable forwarding nodes. The sender node applies the relay suitability measurement to these nodes and improves the efficiency of routing. The proper selection of routers facilitates the scaling of RESP routing to a large network without reducing its routing reliability.

\subsection{Different routing aspects of relay suitability factor}

The main aim of the proposed methodology is to design a scalable and an efficient geographic routing protocol by considering different aspects of routing strategy and efficiently integrating the compensating routing factors. The source selects the neighbors from the preferable forwarding region before sending the data packet. To select the router, the RESP protocol exploits the RElay Suitability (RES) as a metric of communication quality. A sender node selects a neighbor node $\in$ MPFN with high RES to enhance the reliability. If the sender node simply selects a neighbor with a low PER as per the distance metric, the routing delay increases because of the increase in the number of hops to the DN. It limits the scalability of the network to support video streaming in VANETs. Hence, the proposed RESP applies the delay, collision impact, stability, and ETX metrics in weighted division algorithm and selects a high-quality router for video streaming.

\subsection{Forwarding probability and delay metrics}

The most deciding factors of forwarding probability are collision-free network and link stability. The proposed RESP protocol suggests the estimation of link stability according to the 


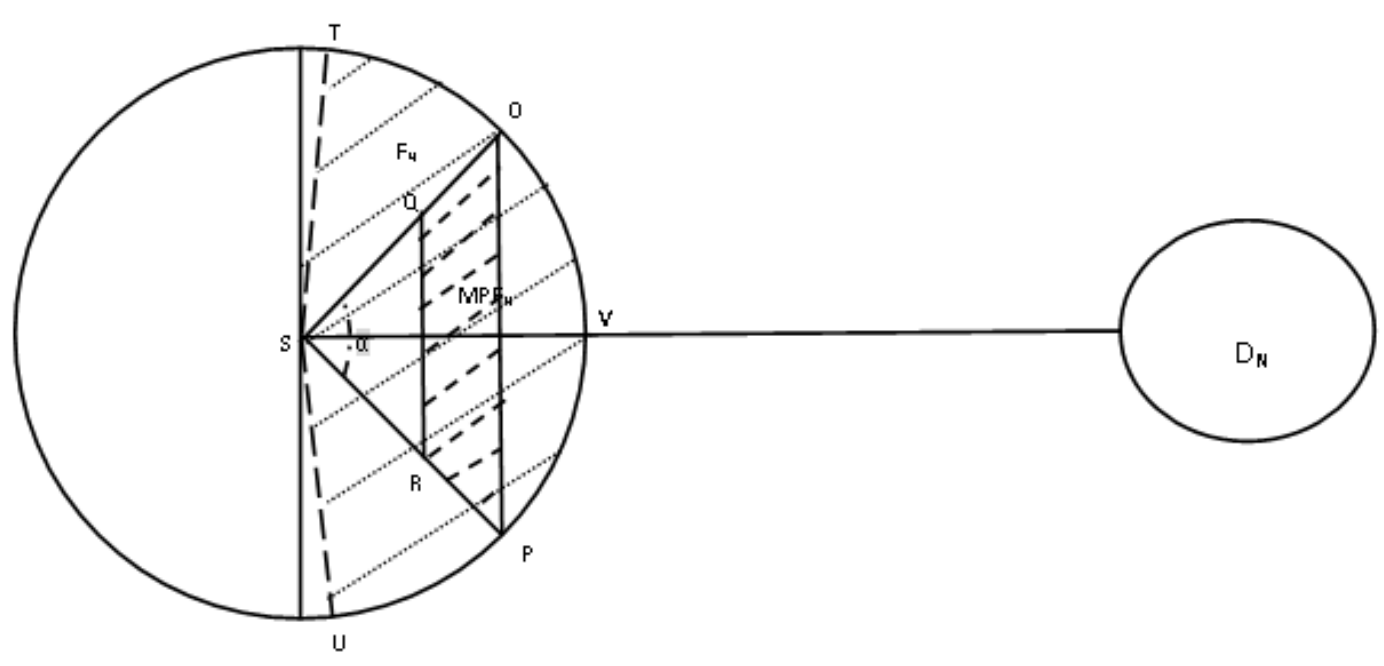

Figure 2: Most preferable forwarding regions

relative velocity of two vehicles and data collision probability using node density. The current works considered node velocity but the use of node velocity alone cannot guarantee the estimation of the future network topology from the relative velocity of two vehicles since their velocity does not depend on their proximity. Based on this, the relative velocity of a node with the neighboring nodes located in the restricted forwarding region was considered in the proposed protocol. If the vehicles move in the same direction at the same speed, their relative velocity is considered as zero, but if they are moving in the same direction with a significant difference in their speed, the relative velocity is considered high. The relative velocity $(\Delta \mathrm{V})$ is used as a primary factor in the determination of relay suitability.

$$
\Delta V_{i}=V_{S N}-V_{i}, \text { Where } i \text { varies from } 1 \text { to }\left|M P F_{N}\right|
$$

Moreover, the packet collision increases with the number of active nodes (the nodes transmitting the packets simultaneously), even as a high traffic to a selected intermediate vehicle can cause both collision and packet loss. Thus, the RESP estimates the probability of packet dropping due to collisions. The packet level lossy network collision model for each link $N_{i, j}$ is taken as the input number of nodes that add the node $\mathrm{j}$ to its corresponding MPFN (NNM) over the total neighbors. Equation 7 showed the estimation of collision probability of node $\mathrm{j}$.

$$
C P_{j}=\frac{\left(\text { Area }_{\text {network }} x N N_{M}\right)}{\left(\text { Total Nodes } x 3.14 x R^{2}\right)}
$$

It considered the network area, total number of nodes, and the communication range of the nodes. Assume that all the nodes know the network area and the number of nodes; when a node is added in the MPFN of most of its neighboring nodes, there is a chance for packet collision. The delay and ETX are essential parameters for video streaming over VANETs and to deliver video files to their destination on time, it is essential to select efficient routing nodes. Considering ETX alone expose the routing protocol to long routing path determination and high latency on routing video frames.

$$
E T X_{i, j}=\frac{1}{\left(P_{i, j} x P_{j, i}\right)}
$$

By applying Equation 8, the ETX value for a communication link $i j$ can be estimated, where $P i j$ and $P j i$ represent the packet delivery ratio of links $i j$ and $j i$, respectively. By using all 
the factors, the proposed RESP estimates the RES value for all the nodes $\in$ MPFN. Moreover, the distance metric-based selection router or the greedy node is not much reliables since the can move out of the communication range anytime. Thus, the distance or ETX metric alone is inefficient for video streaming over VANETs and in the RESP, both metrics were considered as forwarding delay factors.

\subsection{Scalable and QoS data forwarding by applying weighted division algo- rithm}

The proposed RESP considered multiple routing aspects such as the probability of packet dropping due to the collision, ETX, relative velocity, and distance factor. However, an improper integration of these factors tends to compensate each other for their influence on the scalable and efficient router selection for video streaming since the greedy node drops the packet due to mobility and enables the sender node to retransmit the same packet frequently. Moreover, the selection of the shortest link for routing increases video frame delivery delay and video buffering. Thus, the proposed RESP integrates the compensating factors using a weighted division algorithm to improve the performance of a VANET router selection process.

$$
\text { Link Quality }_{i, j}=\left\{\frac{\left[(1-\alpha)\left(1-\frac{\Delta V_{i, j}}{R}\right)\right]+(\alpha)\left(1-C P_{j}\right)}{(1-\beta)(E T X)+(\beta)\left(1-\left[\frac{\text { Dist }_{i, j}}{R}\right]\right.}\right\}
$$

In Equations 8 and 9, $\alpha$ and $\beta$ are the weighting factors, and moreover, both values are in the range of 0 to 1 . The stability and collision probability is the forwarding probability relevant metrics. If the ratio of $\frac{\Delta V_{i, j}}{R}$ is nearly 1 , the link will be disconnected. The factor $C P_{j}$ also creates a negative impact. The weighted addition of factors $1-\left(\frac{\Delta V_{i, j}}{R}\right)$ and $\left(1-C P_{j}\right)$ are taken in the denominator whereas the weighted summation of ETX and geographic advancement are taken in the numerator. The result of a weighted division algorithm represents the quality of the communication link. The node with a high link quality in the MPFN is selected in the packet forwarding. The collision-free links with a better stability significantly mitigate the packet delay and dropping. Thus, the selection of high-quality links ensure a successful delivery of video frames and a significant reduction in video distortion and buffering.

The algorithm 1 illustrates the process of RESP protocol in detail. The processes in RESP protocol are divided into three procedures, such as Most Preferable forwarding region selection, Relay suitability (Link quality) Measurement, and Scalable router selection. Every node that has data packets to forward to the destination executes the aforesaid three procedures to select the scalable router for video streaming. The following flow chart in Figure 3 provides a detailed picture of processes in RESP protocol.

\section{Performance evaluation}

The performance of the proposed RESP was evaluated in an NS2 simulator on a $2000 \mathrm{~m}$ road scenario where the vehicles move in two independent directions. The simulation model imports the roadmap from the SUMO. The maximum velocity of vehicles in each lane was 130 $\mathrm{km} / \mathrm{h}$. In this scenario, the vehicles follow MOVE mobility model for movement. To evaluate the performance of the RESP, it was compared to LBRP [11], LSGR [13] and GPSR [18] under various scenarios by varying the number of vehicles. The agents such as MyTrafficTrace, MyUDP, and MyUDPSink were built inside the NS2 to generate video traffic. The sender forwards the video format of YUV QCIF or YUV CIF to the destination. To support the digitized video transfer, this work utilized the MPEG4 codec to compress the file. The MyTrafficTrace agent fragments 


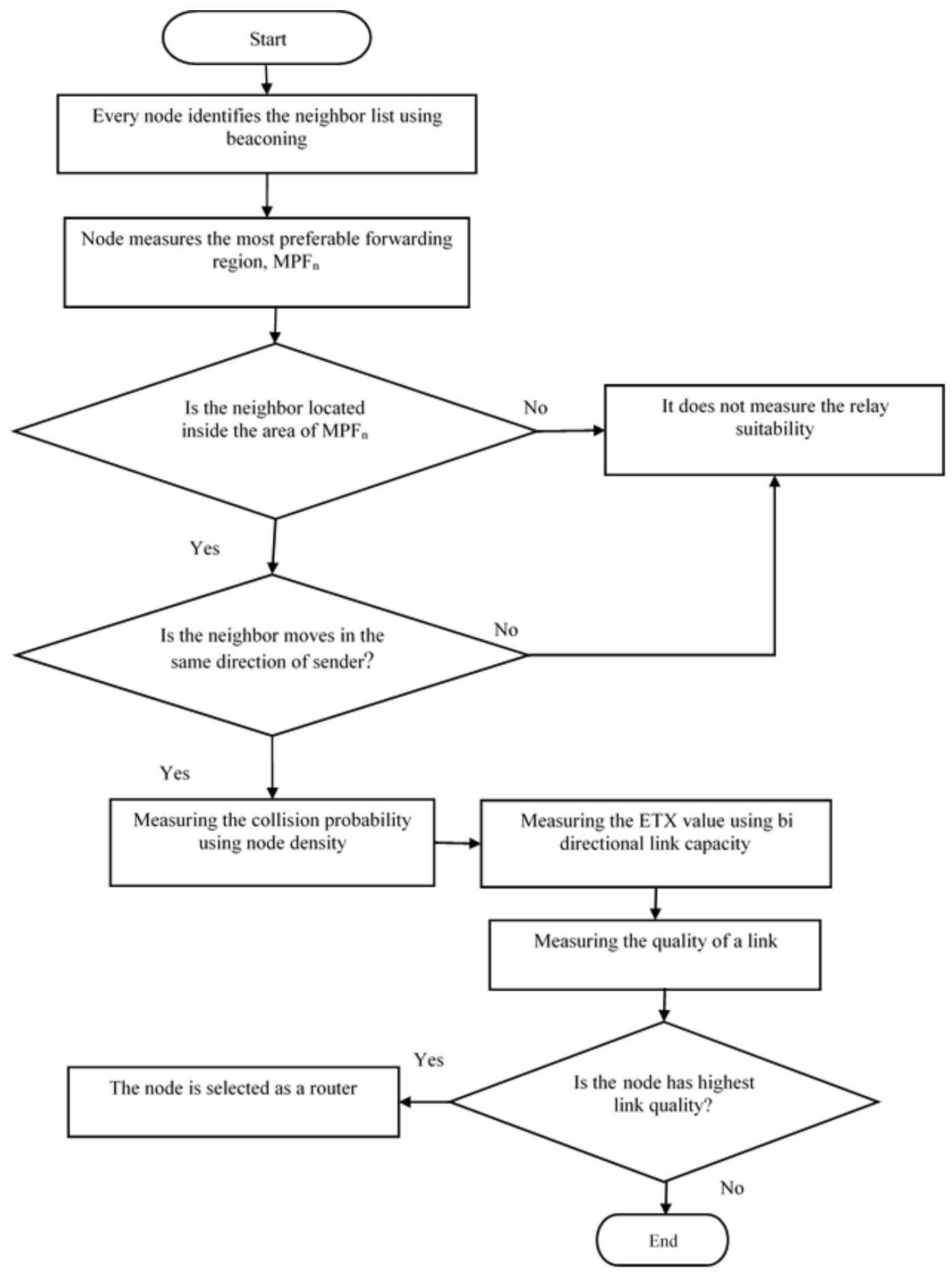

Figure 3: Flow chart of process of RESP protocol 


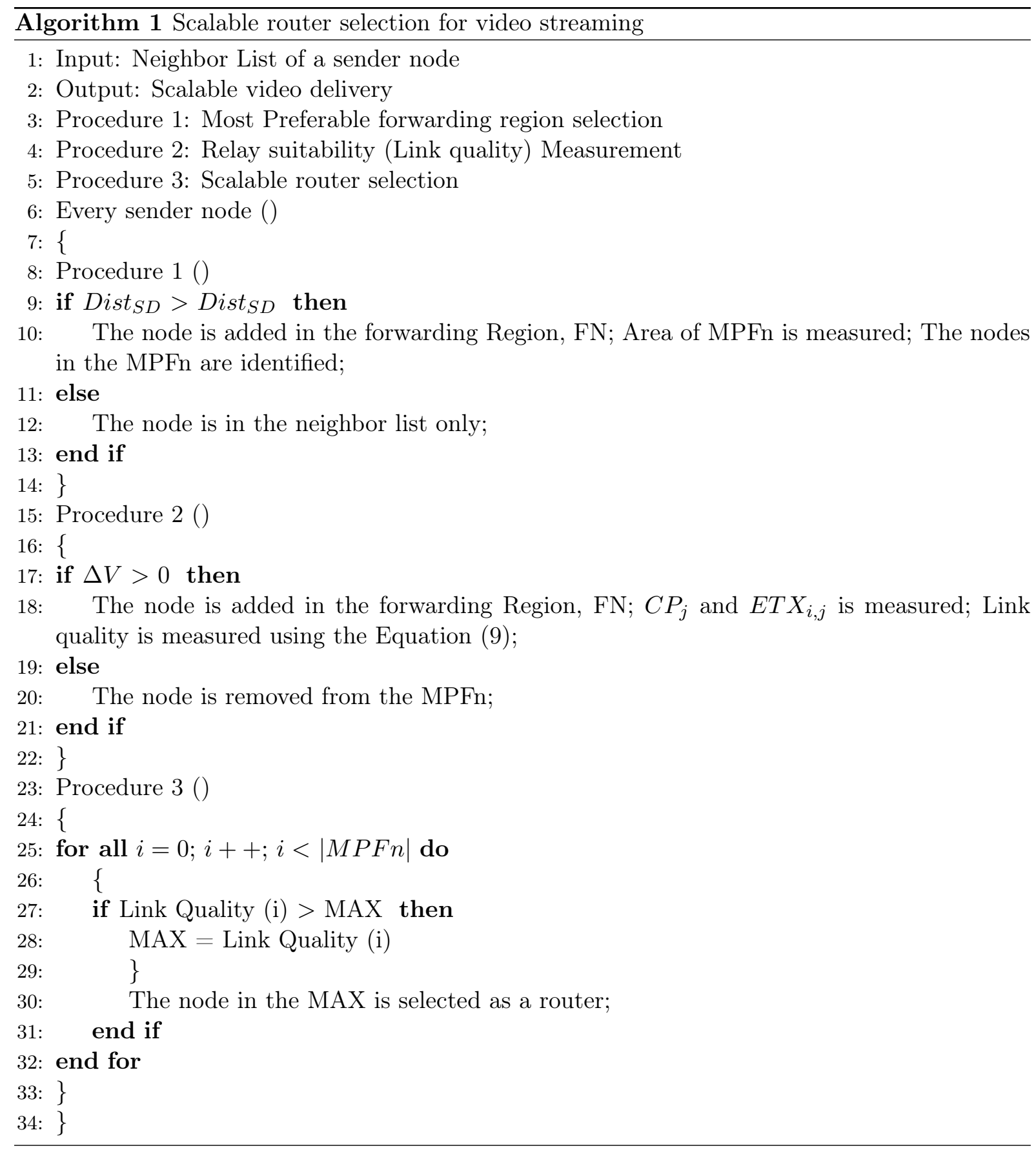


each of the video frames in the compressed file into several segments. It was transmitted using MyUDP and received using MyUDPSink on the receiver side. The sender trace file retains the timestamp, the packet id, and the packet payload size for every UDP packet transmission, and the video trace file holds information about each frame. By utilizing the Tcl script, the network objects were configured for video streams. The video clip used in the simulation was $5.7 \mathrm{MB}$ in size, 30 fps frame rate, with a pixel resolution of 720 X 576 in MPEG format. The simulation parameters are listed in Table 4.

Table 2: Simulation parameters

\begin{tabular}{|c|c|}
\hline SIMULATOR & Network Simulator 2 \\
\hline Average total vehicle & $50-100$ \\
\hline density $($ Veh $/ \mathrm{km})$ & 130 \\
\hline Vehicle max speed $(\mathrm{km} / \mathrm{h})$ & Randomly \\
\hline Area & 2000 X $100 \mathrm{~m} 2$ \\
\hline Communication Range & $250 \mathrm{~m}$ \\
\hline Interface Type & PHY/Wirelessphy \\
\hline MAC Type & IEEE $802.11 \mathrm{p}$ \\
\hline Queue Type & Droptail/Priority Queue \\
\hline Queue Length & 50 Packets \\
\hline Antenna Type & Omni Antenna \\
\hline Routing Protocol & RESP, LBRP, LSGR, and GPSR \\
\hline Transport Agent & MyUDP, And MyUDPSINK \\
\hline Application Agent & QMytraffictrace \\
\hline Frame Rate & 30 Fps \\
\hline Input Video Format & YUV QCIF \\
\hline Simulation Time & $150 \mathrm{~s}$ \\
\hline
\end{tabular}

\section{Performance metric}

The following performance metrics were used to evaluate the performance of scalable and reliable VANET routing under different density scenarios.

- Packet Delivery Ratio (PDR): It is the ratio between the total received packets and the generated

- Delay: It is the time taken to deliver the data from the source to the destination vehicle.

- Overhead : It refers to the total number of control packets involved in the scalable and reliable video transmission process.

- Frame Setback Ratio: The frame setback ratio is the ratio of the number of delayed frames and the number of forwarded frames per second. These were analyzed to show the impact of the routing schemes on the user perception in scenarios with different end-to-end distances and node densities. 


\section{Results and discussion}

Two key performance metrics are shown in Figure 4, where the performance of the proposed protocol was compared to the performance of three other protocols - LBRP, LSGR, and GPSR. In this figure, the validated influence of vehicular traffic density on performance metrics was on the path lifetime and the overall transmission delay for the target content. In Figure 3a, the performance of the constructed path lifetime was compared. Recall that the path lifetime is determined by the link with the minimum lifetime. As can be seen from the figure, the proposed RESP produced the longest path lifetime since it preferred the neighboring vehicle that can maintain a high connectivity performance. Next in importance is that the LSGR showed the second-best path lifetime as a result of the consideration of both link lifetime and one-hop transmission distance information on the protocol design. On the contrary, without the consideration of the link connectivity property, the path tends to be fragile as illustrated in the GPSR and LBRP. Especially for GPSR, the constructed path is the most easily broken path with the largest inter-relay distance.

Figure 4(b) showed the observed delay of the RESP, LBRP, LSGR, and GPSR when the number of nodes was varied from 50 to 100. RESP achieved a very low packet delivery delay for varying nodes. There is a minimal variation in the packet delay between RESP and LBRP. Most of the delivered packets by RESP reached their destination on time, and this leads to a considerable delay in LSGR and GPSR. However, for the RESP, efficient packet transmissions were performed with a highly stable and less congested communication links. The influencing parameters in the RESP were the node stability and network collision. It minimized the com-

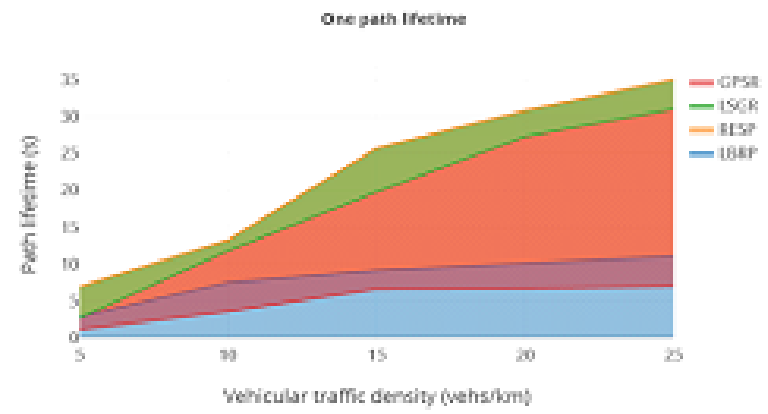

(a)

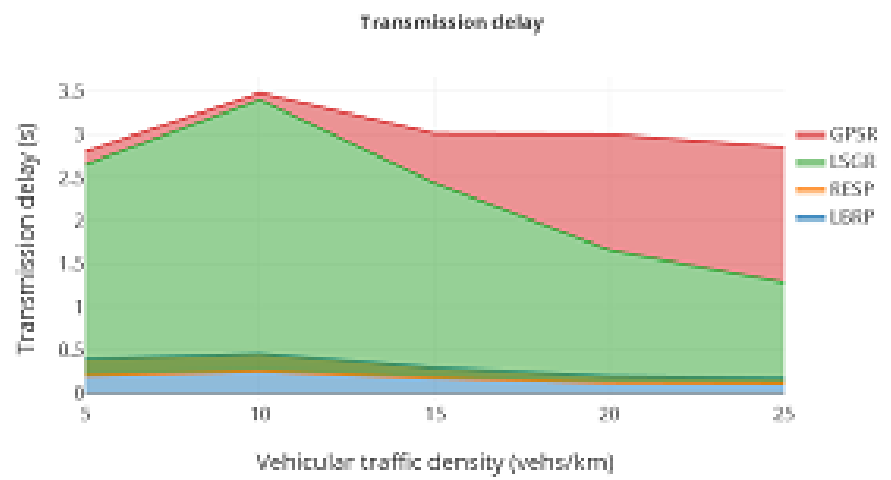

(b)

Figure 4: The three-performance metrics (a) Path lifetime; (b) Transmission delay. Link Lifetime-aware Beacon-less Routing Protocol (LBRP); Link State-aware Geographic Routing (LSGR); Greedy Perimeter Stateless Routing ( GPSR) 
munication delay without degrading the packet delivery ratio. Both RESP and GPSR limits the packet delay in the range of 0.01 to 0.03 seconds. However, LBRP delivers the packets in 0.04 seconds in the low-density network. When the number of nodes increased to 100, the data packets of LBRP were delivered with a delay of 0.02 seconds which was closer to the delay of RESP and GPSR at the same scenario. Even though RESP showed a better performance in terms of delay, the packet delivery ratio was less compared to those of LBRB, LSGR, and GPSR. The path lifetime metrics increases with vehicle speed when the energy factor must be considered in the holding time computation to increase network lifetime.

In Figure 5, the other three performance metrics were shown, where the performance of the proposed RESP was compared to the performance of LBRP, LSGR, and GPSR. In this figure, the validated influence of vehicular traffic density on the performance metrics was in the packet delivery ratio, overhead, and the overall frame setback ratio.

Figure 5 (a) displayed the packet delivery ratio of RESP, LBRP, LSGR, and GPSR when the number of nodes was varied from 50 to 100 . The performance of these solutions was compared for scalability by increasing the vehicle density with the same data traffic. The number of vehicles in the network showed a huge impact on the packet delivery ratio of the 4 protocols. For instance, RESP improved the packet delivery ratio from $81.5 \%$ to $97.8 \%$ when the number of nodes increased from 50 to 100 compared to LBRP, LSGR, and GPSR. RESP achieved the highest delivery ratios because it does not have to select the intermediary vehicles in advance or wait to forward the data packets. Initially, LBRP attained a packet delivery ratio closer to

the RESP. With 70 to 80 nodes, LBRP improved the packet delivery ratio by $33 \%$ more than LSGR. The minimum node density reduced the probability of LBRP failing the communication links between the nodes. However, LSGR required more time to establish the entire path to the destination under a low-density environment and this increased its link breakage probability which resulted in a weaker packet delivery ratio. Beyond 60 nodes, LSGR drastically increased the packet delivery ratio compared to GPSR. The high dense scenario made the construction of the LSGR path faster. With a sufficient number of nodes, RESP improved the packet delivery ratio by $8.4 \%$ and $17.5 \%$ compared to LBRP, LSGR, and GPSR, respectively.

Figure 5(b) showed the overhead of the four protocols under various dense scenarios. As expected, increasing the number of nodes leads to a high overhead due to the increment of control packets. Due to the data transmission interruption in GPSR, the overhead fluctuates with various node density. For instance, LSGR spent 10980 packets to deliver data packets at a low dense scenario while decreasing the overhead to 30205 packets at a high dense scenario. RESP achieved a better result when using the hello packets to generate its neighbor's list and share the velocity information. Compared to the other protocols, RESP attained low overhead at a lower node number, but an increased overhead (from 8960 to 21600 packets) when the node number was increased from 50 to 100. LBRP conducted a receiver-based beaconless routing, however, it transmitted the content request and response packets. The number of these packets are increased compared to the number of packets carrying video contents under a highly dynamic network topology. For instance, LBRP increased the overhead from 15983 to 39875 packets when the number of nodes was increased from 50 to 100 in a high-density scenario but GPSR had the highest overhead rate (starting from 39008 and ending at 79502).

Figure 5(c) showed the result of the frame setback ratio. The delayed data packet is a primary reason behind the FSR value decrement. As expected, the FSR value was increased by all the protocols when the number of nodes was increased from 50 to 100. The RESP considers the relative velocity in link stability measurement rather than the node velocity. It improves the accuracy of node stability measurement and the performance of the RESP. For instance, RESP achieved 0.0014 of FSR when the number of nodes was increased from 50 to 100. Moreover, the number of neighboring nodes having the node identity in their corresponding MPFN list decides 


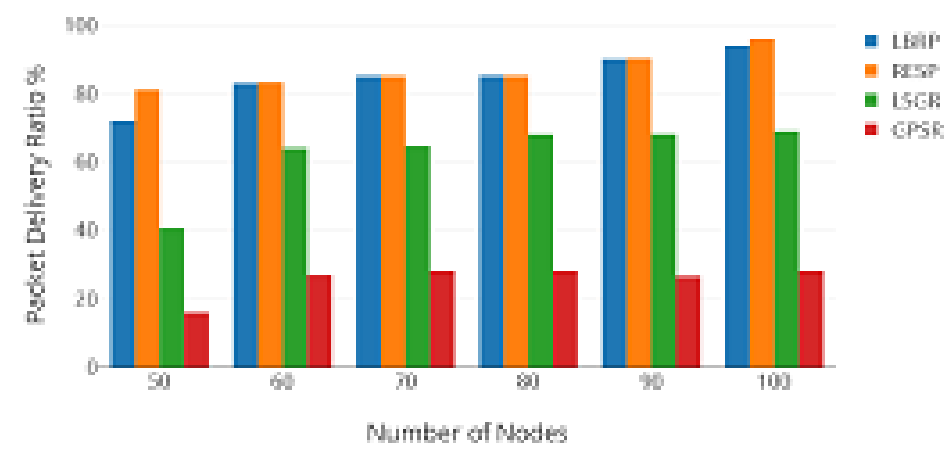

(a)

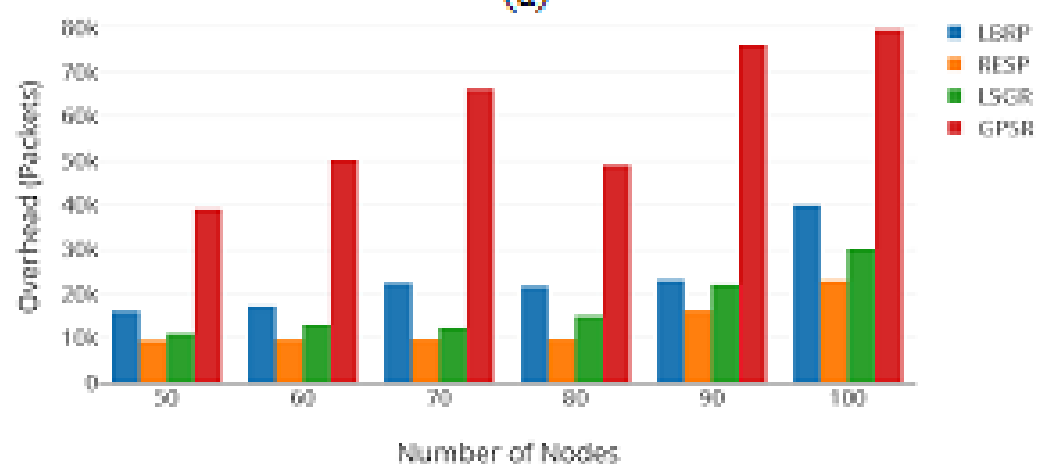

(b)

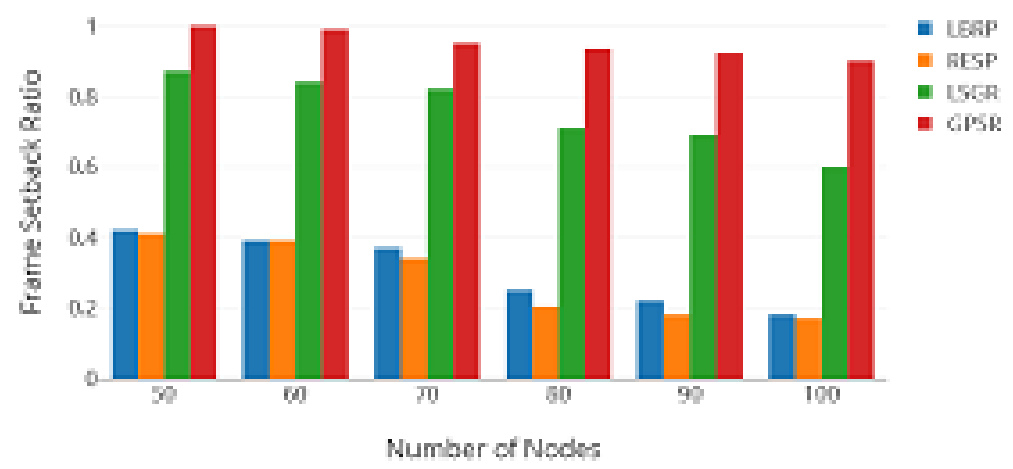

(c)

Figure 5: The three-performance metrics (a) Packet delivery ratio; (b) Overhead; (c) Frame Setback Ratio. Link Lifetime-aware Beacon-less Routing Protocol ( LBRP); Link State-aware Geographic Routing (LSGR); Greedy Perimeter Stateless Routing (GPSR) 
the packet collision at a given node. Considering this scenario, RESP made routing decisions and delivered data packets on time, thereby significantly reducing the frame setback ratio. Compared to LBRP, RESP reduced the frame setback ratio by 0.358 under a high dense scenario.

\section{Conclusion}

This work presented a scalable unicast geographic routing protocol for video streaming in VANETs. The building of a small region at the boundary of the communication range using an offset angle and applying the relay suitability measurement only on those nodes can reduce computational complexities without degrading the performance. In VANET scenarios, vehicle density, vehicle mobility, and destination mobility also reduce the scalability of protocols. The relay suitability measurement includes the forwarding probability and the delay measurement, and this ensures the trade-off between the node geographic advancement towards the destination and the link stability. This work conducted extensive experiments over VANET scenarios where the performance of the proposed and existing unicast routing protocols was compared in terms of scalability by increasing the vehicle density. The simulation results showed improvements in the RESP compared to the existing protocols. RESP was validated via simulation studies and showed to have a greater performance in terms of providing a high PDR and less packet delay with various network density. Moreover, it offered the lowest delay in delivering video frames. It is, therefore, concluded that RESP is a better scalable protocol for delivering video frames on time under a highly dynamic vehicular network topology.

\section{Funding}

The research work has been supported by Universiti Malaysia Pahang (UMP). Grant Id: RDU1703212.

\section{Bibliography}

[1] Ahuja, K.; Khanna, R.; Singh, B. (2011); Real time available-bandwidth estimation (ABE) algorithm based selection in heterogeneous network for wimax and 3G, In Next Generation Mobile Applications, Services and Technologies (NGMAST), IEEE, 2011 5th International Conference on , 169-174. 2011.

[2] Ayoob, A. A.; Su, G.; Wang, D.; Mohammed, M. N.; Hammood, O. A. (2017); Hybrid LTE-VANETs based optimal radio access selection, In International Conference of Reliable Information and Communication Technology, Springer, Cham, 189-200, 2017.

[3] Cabrera, G.; Niklander, S.; Cabrera, E.; Johnson, F. (2016); Solving a distribution network design problem by means of evolutionary algorithms, Studies in Informatics and Control, 25(1), 21-28, 2016.

[4] Chen, Y.; Weng, S.; Guo, W.; Xiong, N. (2016); A Game Theory Algorithm for Intra-Cluster Data Aggregation in a Vehicular Ad Hoc Network, Sensors, 16(2), 245, 2016.

[5] Cunha, F.; Villas, L.; Boukerche, A.; Maia, G.; Viana, A.; Mini, R. A.; Loureiro, A. A. (2016); Data communication in VANETs: Protocols, applications and challenges Ad Hoc Networks, 44, 90-103, 2016. 
[6] De Felice, M.; Cerqueira, E.; Melo, A.; Gerla, M.; Cuomo, F.; Baiocchi, A. (2015); A distributed beaconless routing protocol for real-time video dissemination in multimedia VANETs, Computer Communications, 58, 40-52, 2015.

[7] Eze, E.C.; Zhang, S. J.; Liu, E. J.; Eze, J. C. (2016); Advances in vehicular ad-hoc networks (VANETs): Challenges and road-map for future development, International Journal of Automation and Computing, 13(1), 1-18, 2016.

[8] Hammood, O. A.; Kahar, M. N. M., Mohammed, M. N., Hammood, W. A., Sulaiman, J. (2018) ; The VANET-Solution Approach for Data Packet Forwarding Improvement, Advanced Science Letters, 24(10), 7423-7427, 2018.

[9] Hasan, R. A.; Mohammed, M. A.; Tapus, N.; Hammood, O. A. (2017); A comprehensive study: Ant Colony Optimization (ACO) for facility layout problem, In Networking in Education and Research (RoEduNet), 16th RoEduNet Conference, 1-8, IEEE, 2017.

[10] Hammood, O. A.; Kahar, M. N. M.; Mohammed, M. N. (2017); Enhancement the video quality forwarding Using Receiver-Based Approach (URBA), Vehicular Ad-Hoc Network, In Radar, Antenna, Microwave, Electronics, and Telecommunications (ICRAMET), 2017 International Conference on, IEEE, 64-67, 2017.

[11] Hu, M.; Zhong, Z.; Ni, M.; Baiocchi, A. (2016); Design and analysis of a beacon-less routing protocol for large volume content dissemination in vehicular ad hoc networks, Sensors, 16(11), 1834, 2016.

[12] Husain, A.; Sharma, S. C. (2015); Simulated analysis of location and distance based routing in VANET with IEEE802, Procedia Computer Science, 57, 323-331, 2015.

[13] Li, C.; Wang, L.; He, Y.; Zhao, C.; Lin, H.; Zhu, L. (2014); A link state aware geographic routing protocol for vehicular ad hoc networks, EURASIP Journal on Wireless Communications and Networking, 2014(1), 176, 2014.

[14] Lochert, C.; Mauve, M.; Fubler, H.; Hartenstein, H. (2005); Geographic routing in city scenarios, ACM SIGMOBILE mobile computing and communications review, 9(1), 69-72, 2005 .

[15] Margaria, D.; Falletti, E. (2016); The local integrity approach for urban contexts: Definition and vehicular experimental assessment, Sensors, 16(2), 154, 2016.

[16] Menouar, H.; Lenardi, M.; Filali, F. (2007); Movement prediction-based routing (MOPR) concept for position-based routing in vehicular networks, In Vehicular Technology Conference, 2007. VTC-2007 Fall. 2007 IEEE 66th, IEEE, 2101-2105, 2017.

[17] Mezher, A. M.; Igartua, M. A. (2017); Multimedia Multimetric Map-aware Routing protocol to send video-reporting messages over VANETs in smart cities, IEEE Transactions on Vehicular Technology, 66(12), 10611-10625, 2017.

[18] Mohammed, M. N.; Nahar, A. K.; Abdalla, A. N.; Hammood, O. A. (2017); Peak-to-average power ratio reduction based on optimized phase shift technique, In Communications and Information Technologies (ISCIT), 2017 17th International Symposium on , IEEE, 1-6, 2017.

[19] Orchard, M.; Tang, L.; Saha, B.; Goebel, K.; Vachtsevanos, G. (2010); Risk-sensitive particle-filtering-based prognosis framework for estimation of remaining useful life in energy storage devices, Studies in Informatics and Control, 19(3), 209-218, 2010. 
[20] Quadros, C.; Cerqueira, E.; Santos, A.; Lim, J.; Gerla, M. (2015); Beacon-less video streaming management for VANETs based on QoS and link-quality, In Integrated Network Management (IM), 2015 IFIP/IEEE International Symposium on, EEE, 191-198, 2015.

[21] Quadros, C.; Santos, A.; Gerla, M.; Cerqueira, E. (2016); QoE-driven dissemination of real-time videos over vehicular networks, Computer Communications, 91, 133-147, 2016.

[22] Reyes-Munoz, A.; Domingo, M.; Lopez-Trinidad, M.; Delgado, J. (2016); Integration of body sensor networks and vehicular ad-hoc networks for traffic safety, Sensors, 16(1), 107, 2016.

[23] Saif. A; Moath. M. AL; Ali. H. Al; Hussien. Z. (2014); A comprehensive survey on vehicular ad hoc network, Journal of network and computer applications, 37, 380-392, 2014.

[24] Sharef, B. T.; Alsaqour, R. A.; Ismail, M. (2013); Comparative study of variant positionbased VANET routing protocols Procedia Technology, 11, 532-539, 2013.

[25] Song, C.; Tan, G.; Yu, C. (2017); An Efficient and QoE Supported Multichannel MAC Protocol for Vehicular Ad Hoc Networks Sensors, 17(10), 2293, 2017.

[26] Song, C. (2017); Performance Analysis of the IEEE 802.11 p Multichannel MAC Protocol in Vehicular Ad Hoc Networks, Sensors, 17(12), 2890, 2017.

[27] Spaho, E.; Ikeda, M.; Barolli, L.; Xhafa, F. (2013); Performance comparison of OLSR and AODV protocols in a VANET crossroad scenario, In Information Technology Convergence , Springer, Dordrecht, 37-45, 2013.

[28] Tripp-Barba, C.; Urquiza-Aguiar, L.; Igartua, M. A.; Rebollo-Monedero, D.; de la Cruz Llopis, L. J.; Mezher, A. M.; Aguilar-Calderon, J. A. (2014); A multimetric, map-aware routing protocol for VANETs in urban areas, Sensors, 14(2), 2199-2224, 2014.

[29] Xie, F.; Hua, K. A.; Wang, W.; Ho, Y. H. (2007); Performance study of live video streaming over highway vehicular ad hoc networks, In Vehicular Technology Conference 200\%. VTC2007 Fall. 2007 IEEE 66th , 8(1), 132, 2013.

[30] Xu, S.; Guo, P.; Xu, B.; Zhou, H. (2012); Study on qos of video communication over VANET, In International Conference on Information Computing and Applications, Springer, Berlin, Heidelberg, 730-738, 2012.

[31] Wan, J.; Liu, J.; Shao, Z.; Vasilakos, A. V.; Imran, M.; Zhou, K. (2016); Mobile crowd sensing for traffic prediction in internet of vehicles, Sensors, 16(1), 88, 2016.

[32] Zaimi, I.; Houssaini, Z. S.; Boushaba, A.; Oumsis, M.; Aboutajdine, D. (2016); Vehicular ad-hoc network: Evaluation of QoS and QoE for multimedia application, International Conference on Networked Systems, Springer, Cham, 367-371, 2016.

[33] Zhou, Z.; Dong, M.; Ota, K.; Chang, Z. (2015); Energy-efficient context-aware matching for resource allocation in ultra-dense small cells, IEEE Access, 3(9), 1849-1860, 2015. 